\title{
Tsafon
}

Revue d'études juives du Nord

$81 \mid 2021$

Des synagogues à travers les âges Lieux de prières, lieux d'études et autres fonctions

\section{Présentation du dossier Des synagogues à travers les âges Lieux de prières, lieux d'études et autres fonctions}

Danielle Delmaire

\section{CpenEdition}

Journals

Édition électronique

URL : https://journals.openedition.org/tsafon/3575

DOI : 10.4000/tsafon.3575

ISSN : 2609-6420

Éditeur

Association Jean-Marie Delmaire

Édition imprimée

Date de publication : 1 juillet 2021

Pagination : 7-14

ISSN : 1149-6630

Référence électronique

Danielle Delmaire, «Présentation du dossier Des synagogues à travers les âges Lieux de prières, lieux d'études et autres fonctions », Tsafon [En ligne], 81 | 2021, mis en ligne le 01 juillet 2021, consulté le 15 septembre 2021. URL : http://journals.openedition.org/tsafon/3575 ; DOI : https://doi.org/10.4000/ tsafon.3575 


\section{Dossier}

rassemblé par Emmanuel Friedheim et Danielle Delmaire présenté par Danielle Delmaire

Des synagogues à travers les âges

Lieux de prières, lieux d'études et autres fonctions 
Tsafon 81 
Présenter des " synagogues à travers les âges » et dans toutes les aires géographiques, en moins d'une dizaine d'études, était un pari insoutenable. Nous avons donc prudemment imposé des limites géographiques : le Moyen Orient antique et la France pour les autres périodes avec une excursion vers l'Algérie à l'époque coloniale et après. Quant aux périodes, nous les avons acceptées toutes mais avec un nombre limité de contributions: une seule pour le Moyen Âge et une autre pour l'époque moderne, couvrant toutes deux la Provence, région où les synagogues sont encore bien visibles, deux autres sur les synagogues de la France métropolitaine des $\mathrm{XIX}^{\mathrm{e}}$ et $\mathrm{XX}^{\mathrm{e}}$ siècles et enfin une dernière concerne les synagogues de l'Algérie.

En prenant ce parti de restreindre les investigations à quelques régions, nous nous privions d'informations sur de nombreuses synagogues anciennes et célèbres, comme celles de la Galilée antique (Capharnahum ou Kfar Nahum, Corozin, Beth Alpha pour ne citer que celles-là), celles de l'Europe médiévale avec la fameuse synagogue de Prague, mais encore les magnifiques synagogues de Safed dans la Palestine de l'époque moderne et les non moins belles synagogues de Venise, sans compter les nombreux édifices de l'Europe centrale, tels que le Musée d'Art et d'Histoire Juive les a reconstitués, pour quelques-uns d'entre eux. Quant à l'époque actuelle, les styles varient selon les lieux, pays et continents, depuis les grandes synagogues érigées en France au $\mathrm{XIX}^{\mathrm{e}}$ siècle jusqu'aux petites synagogues qui se dissimulent dans une ruelle (Lunéville) ou qui sont installées dans des maisons dont la façade n'exprime nullement la fonction de lieu de culte (maison de prières de la rue des Prisons à Lille avant l'édification de l'actuelle synagogue que présente Rudy Rigaut). 
Nous avions donc une autre ambition en montant ce dossier. Il ne s'agissait pas de s'intéresser seulement à l'architecture mais d'essayer de déterminer les fonctions de la synagogue : lieu d'assemblée selon les termes grec et hébreu (beth knesset) ou bien lieux destinés à d'autres fonctions ? D'autre part, les quelques exemples retenus permettent-ils de distinguer une évolution dans les habitudes des personnes qui fréquentaient ces synagogues ou au contraire, à travers les âges et les régions, des points communs sont-ils partagés ? Existe-il des pratiques et des styles régionaux ou des pratiques pérennes quels que soient les époques et les lieux?

Dans la Judée et la Galilée de l'époque mishnaïque et talmudique, la maison de l'assemblée (beth haknesset) et la maison d'études (beth hamidrash) partagent-elles les mêmes lieux ? C'est la question à laquelle veut répondre Emmanuel Friedheim. La Tosefta, rappelle cet auteur, précise que dans la synagogue les fidèles lisent, enseignent, étudient mais n'y boivent pas et n'y mangent pas ! Mais, ajoute-t-il, d'autres sources contredisent cette exigence et de conclure «la société juive de l'époque de la Mishna et du Talmud était foncièrement plurivoque ». Si certaines synagogues nous émerveillent, actuellement, avec leurs mosaïques à l'effigie païenne d'Hélios/Sol Invictus comme à Hammat Tibériade, elles étaient « infréquentables pour les Rabbins ». Rites et doctrines variaient donc mais déjà apparaissaient des fonctions autres que la prière : au moins l'étude.

Yaël Escojido confirme cette observation en travaillant sur « La question des Affranchis dans les synagogues de la période du second Temple ». À partir de sources littéraires ou épigraphiques, elle note que la synagogue était bien le lieu de l'assemblée et des études. Si les rabbins exigeaient que l'on y observe une tenue correcte : ni boire, ni manger, d'autres sources montrent que la synagogue était le centre de la vie juive où l'enseignement s'accompagnait de l'exercice de la justice et même pouvait servir d'auberge (inscription de Théodote fils de Ouettènos à Jérusalem, $\mathrm{I}^{\mathrm{er}}$ siècle av. JC à $\mathrm{II}^{\mathrm{e}}$ siècle ap. JC)! Elle constituait « un complexe cultuel, judiciaire avec enseignement». L'auteure conclut: « La synagogue est le centre de l'étude de la Loi juive, on y rend aussi la justice et on y exécute les sentences ».

La ville médiévale de Montpellier possédait une synagogue (peutêtre davantage) qui a disparu. Toutefois, des traces de pratiques rituelles 
trahissent l'existence d'un tel lieu de prières. Michaël Iancu explique que le mikveh médiéval, qui constitue de nos jours une attraction touristique, prouve la diversité de la fonction de la synagogue, qui devait se trouver non loin, selon des sources littéraires et archivistiques. Et, très probablement, les usagers s'adonnaient à la prière et à d'autres activités sans que l'on puisse les définir précisément.

Qu'en est-il pour les synagogues de Provence de l'époque moderne comme les présente Carol Iancu ? L'historien décrit la composition des communautés juives provençales, qui vivaient dans les carrières, et leur environnement social. Dans ce contexte, la synagogue était un édifice complexe avec des salles attenantes réservées à d'autres activités que la prière mais associées à la religion comme la boulangerie pour la fabrication des pains azymes et le mikveh. Les fonctions évoluent et la prière n'est pas la seule activité des fidèles qui y ajoutent d'autre services liés aux usages religieux. Toutefois, à partir de cette époque, on retrouve un agencement commun à toutes les synagogues, : une grande salle pour l'assemblée des hommes où se trouve l'armoire sainte (aron haqodesh) qui abrite les rouleaux de la Torah et l'estrade pour la lecture de ces rouleaux (bema), avec des variantes mineures quant à l'emplacement de la bema et la présence d'un siège pour accueillir Élie.

Avec l'exemple des deux synagogues françaises du XIX ${ }^{\mathrm{e}}$ siècle, celle de La Victoire à Paris et celle de Lille, on constate que l'établissement prend encore de nouvelles fonctions. Les deux édifices sont quasi contemporains: La Victoire fut ouverte en 1874 et la synagogue de Lille fut inaugurée en 1891. Durant ce XIX ${ }^{\mathrm{e}}$ siècle, la situation des juifs en France a bien progressé pour acter leur intégration dans la Nation. Petit à petit, l'égalité citoyenne, accordée aux juifs par la décision de l'Assemblée constituante de 1791, se met en place et devient une réalité même si l'antijudaïsme des catholiques freine quelque peu l'évolution des mœurs et même si, dans ce dernier quart du XIX ${ }^{\mathrm{e}}$ siècle, les acquits de la Révolution semblent ébranlés par l'affaire Dreyfus, point ultime d'une crise antisémite. La marche vers l'égalité a commencé et perdure : s'ils sont menacés, les Français juifs (ou israélites) ont la loi pour eux. Finalement, l'affaire Dreyfus, avec la réhabilitation du capitaine, fut certes trop longue mais elle s'est terminée par le triomphe des valeurs républicaines d'égalité. Et ces changements deviennent visibles dans l'architecture synagogale : à Lille, la synagogue dissimulée dans la maison de la rue des Prisons est remplacée en 1891 par un édifice 
monumental et magnifiquement orné, digne d'une communauté à laquelle la République vient d'attribuer un siège consistorial comme le rappelle Rudy Rigaut.

Ceci est vrai aussi pour La Victoire, sans doute l'une des plus admirée des synagogues de France comme le rapporte un entretien paru dans Actualité juive, $\mathrm{n}^{\circ} 2598$ du 6 mai 2021, p. 13, entre Jonathan Nahmany et David Abitbol qui vient de publier Les synagogues de l'exil aux éditions Messenger, 204 p. «Quelles sont, à vos yeux, les plus belles synagogues d'Europe d'un point de vue architectural ?» demande le journaliste et D. Abitbol de répondre : «La plus emblématique, celle qui se démarque naturellement, est la grande synagogue de la Victoire à Paris ». Selon Claude Nataf, La Victoire est bien emblématique du courant «franco-judaïsme » qui se manifeste par l'intégration dans la société française de la bourgeoisie juive et plus généralement par "l'intégration des Juifs de France dans la Nation et l'égalité entre les cultes ». Lieu où se déroulent les grandes cérémonies patriotiques et où sont célébrés les événements marquants de la communauté parisienne et nationale, la synagogue n'est plus seulement un lieu de prières. La synagogue de la ville de Lille, siège d'un consistoire régional et capitale départementale et régionale, joue le même rôle lorsque le préfet, la maire ou les représentants de la municipalité, les personnalités civiles et militaires participent à des cérémonies officielles qui ne relèvent pas de la religion. Dès lors que les juifs de France sont devenus des citoyens à part entière, une nouvelle fonction est dévolue à la synagogue relevant du politique et de l'intégration sociale. La mutation est sensible dès le XIX siècle lorsque les autorités politiques assistent à l'inauguration de ces deux synagogues.

Autre fonction attribuée à la synagogue à partir du $\mathrm{XX}^{\mathrm{e}}$ siècle : la mémoire. Rudy Rigaut rappelle les ravages des deux guerres mondiales lorsque Lille était occupée. Suite à une explosion accidentelle pour la Première Guerre mondiale et à des détériorations volontaires antisémites pour la Seconde Guerre, la synagogue de Lille doit être restaurée après chacun de ces conflits. Mais la mémoire se porte surtout sur le souvenir des disparus. À Paris comme à Lille, et dans de très nombreuses synagogues, des plaques remémorent le sacrifice des poilus juifs - à Paris, cette mémoire associe l'ami des juifs Charles Péguy à la demande de son épouse - puis laissent une trace des disparus de la déportation, des 
victimes de « la barbarie nazie » comme il est souvent rappelé. Dès lors, la mémoire est présente dans les synagogues au même titre que les prières et l'étude.

Ces nouvelles fonctions de la synagogue apparues du fait de l'histoire ne remplacent pas les autres attributions de la maison de l'assemblée. À Paris et plus modestement à Lille, des salles annexes servent pour d'autres activités : une salle d'étude, une bibliothèque, une cuisine qui permet des goûters voire des repas cashers pour des fêtes communautaires ou des célébrations familiales (bar mitsvah, mariages, kiddoush etc.)

En Algérie, l'évolution est calquée sur ce qui se passe en métropole. Tant que le pays n'est pas encore la colonie française, les synagogues sont des lieux de culte « invisibles » écrit Valérie Assan. Le statut de dhimmi subi par les juifs leur impose modestie et discrétion tout comme les catholiques exigeaient une certaine retenue de la part des juifs dans le royaume de France. Les édifices religieux des juifs ne devaient pas dépasser en volume et en beauté là les églises et ici les mosquées. Avec la colonisation, l'organisation consistoriale s'impose dans le pays et les conditions de vie des juifs s'améliorent surtout avec le décret Crémieux qui, en 1870, leur accorde la citoyenneté française. Les nouvelles synagogues, si elles conservent un style parfois particulier, s'occidentalisent et expriment, tout comme en métropole, la fidélité à la Nation. L'émancipation et l'intégration se lisent dans l'architecture des lieux de culte juifs. Puis vient l'indépendance et la fuite des juifs d'Algérie vers la métropole ou vers Israël. Les synagogues se vident et finissent par être effacées. La mémoire leur échappe et elles ne sont souvent que souvenir.

Nous pouvons conclure ce dossier par quelques remarques tout en affichant la prudence car les exemples sont trop peu nombreux pour que l'analyse soit irréfutable.

Remarquons une constante à travers les âges: la maison de l'assemblée est aussi une maison d'études : malgré des rabbins rétifs le beth haknesset sert aussi de beth hamidrash. La trace en est conservée jusqu'à nos jours dans les expressions qui servent à désigner la synagogue: schule dans la France du nord ou scola dans la France provençale. De même, puisque l'appartenance à une communauté reste forte, il faut accroître les fonctions de la synagogue avec un mikveh pour 
les bains rituels, une boulangerie ou une cuisine pour respecter la fabrication des pains azymes et la casherout. Quant à l'agencement de l'édifice, il semble immuable depuis l'Antiquité : réserver un espace particulier pour conserver les rouleaux de la Torah et en accorder un autre pour leur lecture. La séparation entre l'espace consacré aux hommes et celui dévolu aux femmes reste la norme. Il serait intéressant de regarder comment les communautés libérales réorganisent, de nos jours, la répartition spatiale entre les hommes et les femmes.

La seule nouveauté récente vient de la nécessité ressentie partout en France, et probablement en d'autres endroits en Europe, d'adjoindre à toutes ces fonctions une place importante à la mémoire qui marque, en un premier temps, l'attachement patriotique des juifs de France à leur patrie puis, en un second temps, qui prend en charge la douleur des familles de disparus et qui dénonce la barbarie de la persécution. 